\title{
Participação especial: análise de determinantes e impactos econômicos em projetos marítimos de produção de petróleo
}

\author{
Estela Terumi Massudaa,*, Saul Barisnik Suslick ${ }^{\text {tb }}$, \\ Gabriel Alves da Costa Lima ${ }^{c}$ \\ a,*estela.massuda@gmail.com, UNICAMP, Brasil \\ tbln memoriam, UNICAMP, Brasil \\ 'gabriel@dep.fem.unicamp.br, UNICAMP, Brasil
}

\begin{abstract}
Resumo
A Participação Especial captura parte da renda extraordinária de campos petrolíferos com grande volume de produção, a exemplo do Pré-sal ou campos maduros da Bacia de Campos. Considerando a complexidade desta participação governamental, este artigo analisa seus determinantes e impactos em projetos de petróleo em diferentes cenários: produção isolada com um módulo e lavra com mais módulos. A metodologia inclui elaboração de fluxos de caixas dos projetos e estimativa do VPL, utilizando simulações estocásticas. Os resultados obtidos dos modelos de simulação indicaram a existência de ineficiência/ distorções causadas pela Participação Especial: tanto na sua forma de incidência (alíquotas), como na sua base de cálculo que se manifestam em campos gigantes de petróleo, com diferentes características (até um bilhão de barris - limite utilizado no estudo) e campos nos quais ocorre adição de reservas, pois o segundo módulo é onerado, podendo comprometer a viabilidade econômica deste projeto, conforme nível de preços praticados.
\end{abstract}

Palavras-chave

Participação especial. Petróleo. Viabilidade econômica. Projeto. Metamodelagem.

\section{Introdução}

Os países competem por investimentos baseados na atratividade proporcionada pela prospectividade da base de seus recursos petrolíferos, sistemas regulatórios, regimes fiscais e custos associados (SUSLICK, 2001). A prospectividade das bacias geológicas e os custos são determinantes, de elevada complexidade e de difícil controle. Os sistemas fiscais e regulatórios representam fatores importantes para a dinâmica da exploração e produção de petróleo e, além disso, possuem impactos na atração de investimentos, desenvolvimento tecnológico, entre outros.

Desde a implantação do sistema regulatório em petróleo e gás natural no Brasil por intermédio da Lei do Petróleo (Lei n. ${ }^{\circ}$ 9.478/97) (BRASIL, 1997), observa-se a ocorrência de mudanças significativas no cenário de E\&P (exploração e produção) no país. Um dos principais indicadores refere-se às arrecadações geradas pelas participações governamentais que apontam valores significativos, distribuindo um percentual importante da riqueza gerada na atividade extrativa do petróleo entre os diversos níveis do setor público (União, Estados e Municípios), bem como aplicações nas áreas de P\&D (pesquisa e desenvolvimento) do segmento petrolífero e de meio ambiente. Além disso, em 2008 constatou-se um envolvimento de mais 50 empresas nas etapas de exploração e produção de petróleo.

0 sistema fiscal brasileiro possui duas formas de apropriação da renda petrolífera (CTN, 1966 e Lei n. ${ }^{\circ}$ 9.478/97): os tributos (aplicados às atividades econômicas) e as participações governamentais 
(aplicadas somente à atividade de exploração, desenvolvimento e produção de petróleo e gás natural) ${ }^{1}$. A partir da Lei do Petróleo foram criadas as participações governamentais que incluem quatro modalidades: os royalties, o bônus de assinatura, a retenção de área e a participação especial, a qual se constitui no principal foco deste trabalho.

A participação especial (PE) tem um peso importante por ser um instrumento de destaque na arrecadação do governo, na apropriação de parte da renda proveniente da produção de petróleo e gás natural, nos casos de grande volume de produção ou de grande rentabilidade. Essa importância pode ser percebida nos dados de arrecadação da ANP (AGÊNCIA NACIONAL DO PETRÓLEO, GÁS NATURAL E BIOCOMBUSTÍVEIS, 2008), que demonstraram que entre 2000 e 2008 o montante da PE foi de $\mathrm{R} \$$ 50,2 bilhões, representando aproximadamente $50 \%$ do total arrecadado em participações governamentais, valor equiparado ao dos royalties.

Uma característica importante dos tributos e participações governamentais é que eles devem ser neutros, ou seja, não podem gerar distorções na decisão de investir em novos projetos, especialmente nas regiões com grandes reservas petrolíferas e custos elevados. No entanto, tal característica nem sempre se verifica na prática.

Tributos semelhantes à participação especial são utilizados por diversos países visando capturar uma parte da renda econômica gerada pelos campos de petróleo em períodos de preços elevados e/ou alta produtividade. A teoria econômica mostra que a tributação ótima deve incidir sobre a renda econômica gerada por um fator de produção. A renda econômica, por sua vez, é definida como o excesso ganho por um fator de produção sobre o necessário, para induzir, fazer seu trabalho ou a renda em excesso sobre o valor necessário para manter um fator de produção em sua ocupação atual, permitindo combinar a relação entre risco e retorno. Gamponia e Mendelsohn (1985), Fraser (2002), Tilton (2004) e Nakhle (2007) realizaram diferentes estudos envolvendo níveis ótimos de taxação adequados na produção de recursos não renováveis visando estabelecer um equilíbrio racional entre o estado e as empresas, apontando as vantagens e as desvantagens para ambos protagonistas.

A tributação e o impacto das participações governamentais e suas relações com os projetos de

\footnotetext{
'A fatia do governo (ou Government Take-GT, na denominação internacional) é composta no Brasil pelos tributos e participações governamentais. Os tributos incluem os impostos, taxas e contribuições (Brasil, Código Tributário Nacional 1966) e as participações governamentais incluem a participação especial, royalties, bônus de assinatura e retenção de área (Lei n. ${ }^{\circ} 478 / 97$ ).
}

exploração e produção de petróleo e gás natural foram abordados por Barbosa e Bastos (2001), Pereira (2004), Gandra (2006) e Rocha, Ribeiro e Marques (2007). A particularidade e a principal motivação neste trabalho é compreender a dinâmica da PE nas seguintes condições: 1) onde há diferentes cenários de tamanhos de reserva e preços; 2) onde ocorre a adição de reserva (ou produção em módulos), que são situações bastante frequentes na maioria dos grandes projetos de petróleo em águas profundas e ultraprofundas no Brasil. Outra motivação para a análise do impacto da PE refere-se às descobertas recentes realizadas pela Petrobras em rochas denominadas de pré-sal, em horizontes profundos da bacia de Santos. Essa nova fronteira exploratória vem ensejando novos estudos e perspectivas de mudanças por parte dos organismos governamentais no sistema regulatório e consequentemente na forma de incidência da PE.

0 trabalho está estruturado em quatro seções e a presente introdução. A primeira seção apresenta uma descrição sucinta sobre a participação especial no Brasil em projetos de produção de petróleo. A segunda seção traz a descrição da metodologia para a realização do trabalho. A aplicação da metodologia em diversos cenários de preços e reservas é descrita na terceira seção. A quarta seção mostra uma metamodelagem entre as variáveis VPL (valor presente líquido) e PE e seus determinantes, seguido das considerações finais do trabalho.

\section{Participação especial em projetos de produção de petróleo}

No modelo adotado no Brasil, o Estado é o detentor dos recursos minerais, mas transfere por meio de um contrato de concessão os direitos de exploração e produção para terceiros, inclusive os respectivos frutos, remunerando-se por intermédio de tributos e participações governamentais exigíveis do concessionário. Dentre as participações governamentais, a participação especial (PE) constitui compensação financeira extraordinária devida pelos concessionários de produção de petróleo ou gás natural nos casos de grande volume de produção ou de grande rentabilidade e será paga, com relação a cada campo de uma dada área de concessão, a partir do trimestre em que ocorrer a data de início da respectiva produção (Decreto ANP n. ${ }^{\circ}$ 2.705/98 - Artigo 21ㅇ) (BRASIL, 1998). Embora na legislação esteja definido que os fatos geradores da PE são grande volume de produção e grande rentabilidade, na prática o que vem sendo utilizado é somente o primeiro elemento, que será detalhado mais adiante. 
As alíquotas da PE dependem de três fatores básicos: faixa de produção, tempo a partir do início da produção do campo e profundidade de lâmina d'água do reservatório. As alíquotas são progressivas $(0,10,20,30,35,40 \%)$ sobre a receita líquida da produção do campo no período-base, de acordo com a sua localização (lavra em terra, águas rasas até $400 \mathrm{~m}$ e águas profundas acima de $400 \mathrm{~m}$ de lâmina d'água), o número de anos de produção $\left(1^{\circ}\right.$, $2^{\circ}, 3^{\circ}$ ou $4^{\circ}$ ano em diante) e os respectivos volumes de produção fiscalizada de petróleo e gás natural, convertidos em volume de petróleo equivalente, de acordo com o procedimento estabelecido no art. 12 da Portaria ANP n. ${ }^{\circ}$ 10/99 (BRASIL, 1999).

Como mencionado anteriormente, o fato gerador da participação especial (PE) é a produção ${ }^{2}$ ou a elevada rentabilidade. 0 início da produção é a data de referência para fins de contabilização da PE. Ocorre que quando o campo tem reserva muito grande, ele tende a ser "modularizado", ou seja, desenvolvido por partes (módulos), devido aos elevados valores de investimentos necessários e os riscos associados a cada fase.

No caso em que ocorrer a produção em módulos (vários projetos subsequentes, em um mesmo campo, oriundos da mesma concessão) ou quando há adição de reservas em um mesmo campo $^{3}$ que já estava em produção, a participação especial será cobrada considerando-se a data de início da produção do primeiro módulo. É quando ocorre a distorção, pois como o tempo ("relógio") da PE começa contar a partir do início da produção do primeiro módulo, quando o segundo módulo (novo projeto complementar) entra em produção, a PE considera como se fosse um único projeto acumulado de produção e, portanto, já inicia "pagando" a alíquota maior. Na simulação realizada a seguir, a alíquota da PE passaria de $0 \%$ até $40 \%$, somente pelo fato da adição sobre outra reserva já em produção.

A Figura 1 mostra o fluxo de eventos relacionados à forma da incidência da PE para um projeto com dois módulos, com produção inicial de $250 \mathrm{MMbbl}$ (milhões de barris) e adição do segundo módulo com mesmo volume de reserva recuperável de 250 MMbbl.

\footnotetext{
${ }^{2} \mathrm{Na}$ prática, conforme mencionado, a forma de incidência da PE enfatiza a grande produção como fato gerador para a cobrança da PE. No entanto, os reservatórios de petróleo apresentam propriedades heterôgeneas (porosidade, permeabilidade, qualidade do óleo, entre outras), sistemas de produção distintos e essas características ocasionam assimetrias entre indicadores de rentabilidade (custos e seus determinantes associados) e volumes de produção. Nesse sentido, observa-se de maneira frequente que nem sempre grande produção significa grande rentabilidade e vice-versa.

${ }^{3}$ Novos poços e atividades de desenvolvimento da reserva podem indicar volumes adicionais de hidrocarbonetos.
}

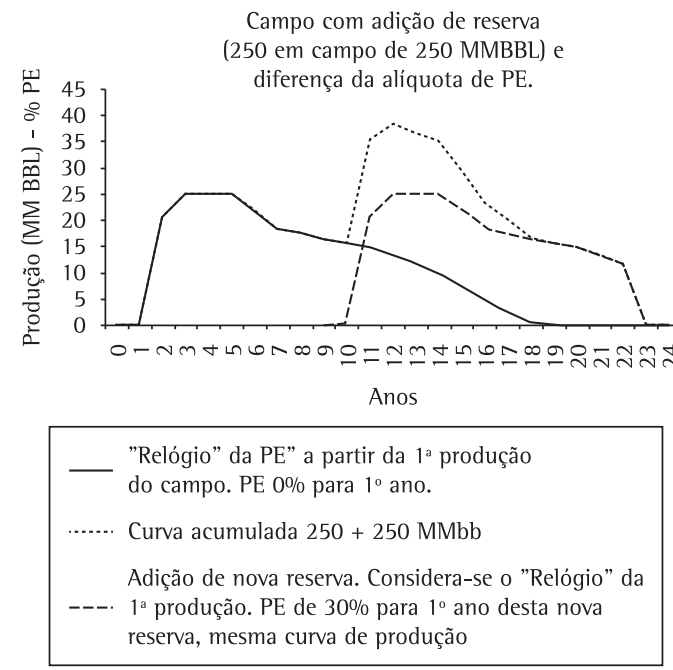

Figura 1. Comportamento incidência da PE para campos com adição de reservas / produção em módulos. Fonte: Massuda (2008).

Na Figura 1 são apresentados dois casos: i) a incidência da PE no caso em que a reserva do campo for 250 milhões de barris; e ii) a incidência da PE no caso de um campo onde inicialmente a reserva é 250 milhões de barris e depois são adicionados mais 250 milhões de barris (total de 500 milhões de barris) e duração de 24 anos.

Para entender o comportamento da PE em diferentes situações, suponhamos uma área de concessão que possui um campo que será desenvolvido em três módulos: A, B e C. A produção do campo A teve início em 2008. A produção do módulo B começa em 2010 e a produção do módulo C em 2013. Neste caso, inicialmente a PE incide sobre a renda decorrente da produção do campo A. Depois, com o início da produção do módulo $\mathrm{B}$, a PE será aplicada à renda decorrente da soma da produção de A e B. Mas, em 2010 o campo A já possui dois anos e paga uma alíquota mais alta. A produção do módulo B, por sua vez, começa em 2010 e deveria pagar alíquotas mais baixas. Entretanto, uma vez que os módulos A e B fazem parte da mesma área de concessão, para efeitos de PE o início da produção se dá com o start-up (início) da produção do módulo A e, assim, a base de cálculo da PE a partir de 2010 é a soma da produção de A e B. Dessa maneira, o valor da PE devido pelo concessionário em 2010 é mais elevado. Ao chegar em 2013 há início da produção do módulo C. Sua alíquota deveria ser baixa no primeiro ano. Porém, como $C$ se encontra na mesma área de concessão de $\mathrm{A}$ e $\mathrm{B}$, a alíquota considera a soma da produção somada de $\mathrm{A}, \mathrm{B}$ e $\mathrm{C}$, e considera que o início da 
produção é a do módulo A. 0 resultado é que à medida que se adicionam mais módulos, maior será a fatia dos fluxos de caixas capturados pela PE.

Sobre a base de cálculo da PE: embora a base de cálculo da PE seja a receita líquida da produção trimestral de óleo e gás, há diversos itens que podem ser deduzidos para a apuração da receita líquida tributável, tais como diversos custos da atividade de E\&P que ocorrem na zona de influência (ring fence) em torno da área de concessão. As demais deduções da receita bruta permitidas contemplam: participações governamentais (royalties, bônus de assinatura, pagamento pela ocupação e retenção da área, $1 \%$ do investimento em pesquisa $\mathrm{e}$ desenvolvimento) entre outras deduções podem ser encontradas nas Portarias ANP n. ${ }^{\circ}$ 10/99 e 102/99 (AGÊNCIA NACIONAL DO PETRÓLEO, GÁS NATURAL E BIOCOMBUSTIVEEIS, 2009).

Os recursos arrecadados pelo Estado na forma de participações são distribuídos entre as diversas esferas de governos na forma determinada pela legislação em vigor (AGÊNCIA NACIONAL DO PETRÓLEO, GÁS NATURAL E BIOCOMBUSTÍVEIS, 2009). Além disso, por força dos contratos de concessão as empresas concessionárias são obrigadas a investirem um valor equivalente a $1 \%$ da receita proveniente da $\mathrm{PE}$, do qual até 50\% poderão ser aplicados em despesas qualificadas como pesquisa e desenvolvimento, executadas em instalações próprias da empresa, e o restante deve ser aplicado em universidades e institutos de pesquisa nacionais. Maiores detalhes sobre essa importante contribuição da PE podem ser encontrados em ANP (AGÊNCIA NACIONAL DO PETRÓLEO, GÁS NATURAL E BIOCOMBUSTIVEIS, 2008).

\section{Metodologia para análise do impacto da participação especial no VPL do projeto}

A metodologia proposta para estudar os impactos da PE envolve as seguintes etapas: 1) elaboração dos fluxos de caixa dos projetos; 2) modelagem de incerteza de variáveis como preço, custo etc; 3) realização de uma regressão multivariada entre o VPL (valor presente líquido) e variáveis mais críticas. As principais premissas dos fluxos de caixa dos projetos encontram-se descritos na Tabela 1.

As premissas da Tabela 1 são típicas para muitos campos de óleo e gás que atualmente se encontram em produção nas bacias marítimas sedimentares do Brasil. Uma vez que não há certeza sobre os valores reais das premissas da Tabela 1 , são adotados os seguintes cenários:

- Volumes das reservas: 250, 500, 750 e 1000 milhões de barris, os quais são cenários típicos de reservas para ambientes de águas profundas e ultraprofundas.
Tabela 1. Principais premissas econômicas e fiscais empregadas no fluxo de caixa dos projetos.

\begin{tabular}{|c|c|}
\hline Variáveis & Caso-base \\
\hline Tempo de produção do campo & 22 anos \\
\hline Preço do óleo - Brent & $36,00 \mathrm{US} \$ / \mathrm{bbl}$ \\
\hline Campo/critério de PE & > 400 m de lâmina d'água \\
\hline Qualidade do óleo & $35^{\circ} \mathrm{APl}$ \\
\hline Taxa mínima de atratividade & 10\% а.а. \\
\hline Taxa de câmbio & 2,20 R\$/US\$ \\
\hline Inflação & $4,0 \%$ a.a. \\
\hline Royalty & $10 \%$ \\
\hline Bônus de assinatura & US\$ 5 milhões \\
\hline Retenção de área & $882,12 \mathrm{R} \$ / \mathrm{km}^{2} /$ ano \\
\hline Alíquota de Imposto de Renda & $25 \%$ \\
\hline Alíquota de Contribuição Social & $9 \%$ \\
\hline Alíquota de Cofins & $0,65 \%$ \\
\hline Alíquota de PIS & $3 \%$ \\
\hline Alíquota de ICMS & $18 \%$ \\
\hline Alíquota de ISS & $5 \%$ \\
\hline Alíquota de 11 & $15 \%$ \\
\hline Alíquota de IPI & $7 \%$ \\
\hline Capex - Investimentos & $5,71 \mathrm{US} \$ / \mathrm{bbl}$ \\
\hline Opex - Custos operacionais fixos & 1,50\% do Capex ao ano \\
\hline $\begin{array}{c}\text { Opex - Custos operacionais } \\
\text { variáveis }\end{array}$ & 2,45 US\$/bbl \\
\hline Abandono & $0,25 \mathrm{US} \$ / \mathrm{bbl}$ \\
\hline Depreciação & 10 anos \\
\hline Área de retenção & $250 \mathrm{~km}^{2}$ \\
\hline Repetro(*) & Não \\
\hline
\end{tabular}

Observacões e fontes: Os valores de Capex (investimentos) e Opex (custos operacionais) foram estimados a partir de dados da literatura: Oil and Gás Journal (diversos), Brasil Energia (diversos) e Petrobras (diversos). (*) Os benefícios fiscais proporcionados pelo Repetro não foram incluídos nas simulações.

- Caracterização do desenvolvimento dos campos em módulos: A partir dos cenários de volumes de reservas definidos 250, 500, 750 e $1000 \mathrm{MMbbl}$ (milhões de barris) adicionou-se um módulo de 250 MMbbl para cada volume de reservas inferiores a 1 bilhão de barris, respectivamente. Dessa maneira, por exemplo, o campo do tipo $500+250 \mathrm{MMbbl}$ significa que há dois módulos que entraram em produção numa mesma concessão, sendo que o $1^{\circ}$ projeto foi de $500 \mathrm{MMbbl}$ e, depois, foi adicionado o $2^{\circ}$ projeto com mais $250 \mathrm{MMbbl}$ que começou a produzir em um período posterior, no mesmo campo, totalizando $750 \mathrm{MMbbl}$ de reserva.

- Cenário de preço de mercado: os preços considerados foram iguais a 36,50 e $70 \mathrm{US} \$ / \mathrm{bbl}^{4}$.

\footnotetext{
${ }^{4}$ Os preços foram estimados a partir da série de preços históricos mensais entre 1998 e 2008 do U.S. Energy Information Administration (ElA) e adotados como referência para montagem dos fluxos de caixa para a simulação estocástica.
} 
Dessa forma, tem-se sete cenários de produção (reservas) e três cenários de preços, totalizando 21 cenários analisados no presente trabalho. Ressalta-se que na análise dos projetos a lâmina d'água foi definida como acima de $400 \mathrm{~m}$, e a estimativa da qualidade do óleo foi baseada no perfil médio de viscosidade e densidade dos valores de óleos encontrados nos campos brasileiros. A taxa mínima de atratividade (TMA) foi estimada a partir de dados coletados nos relatórios de empresas como a Petrobras e dados existentes na literatura. Os demais dados foram extraídos do simulador do LAGE - Laboratório de Análise Geoeconômica da Unicamp (www.ige.unicamp.br/ lage).

A partir das premissas definidas anteriormente, foram calculados os indicadores econômicos dos 21 fluxos de caixa realizados para os 21 cenários. Em seguida, foram estimados o VPL e outros indicadores dos fluxos de caixa do governo e da empresa. Na Tabela 2 encontra-se uma descrição dos principais cenários analisados para se verificar o impacto da PE no VPL.

As informações presentes na Tabela 2 devem ser interpretadas da seguinte forma: uma reserva de 500 milhões de reserva pode ser desenvolvida de uma única vez (cenário 1) ou em partes, podendo ser lavrada considerando-se inicialmente 250 milhões e, depois, adicionando-se 250 milhões (cenário 5). 0 mesmo raciocínio deve ser empregado para as demais reservas.

Além de se verificar o impacto decorrente do regime fiscal, foram analisadas as variáveis incertas que mais causam impactos no VPL e PE. Para isso, inicialmente, foram selecionadas as seguintes variáveis incertas: preço do óleo, custo de investimento de capital, custo operacional e qualidade do óleo. A seguir, o modelo de regressão linear que será utilizado:

$P E i=\beta_{1}+\beta_{2} P_{i}+\beta_{3} C_{i}+\beta_{4} O_{i}+\beta_{5} Q_{i}+\varepsilon_{i}$

VPLi $=\beta_{1}+\beta_{2} P_{i}+\beta_{3} C_{i}+\beta_{4} O_{i}+\beta_{5} Q_{i}+\varepsilon_{i}$

onde:

- $P E=$ participação especial;

- $V P L=$ valor presente líquido;

- $P=$ preço do petróleo;

- $C=$ é o valor do Capex (investimento) por barril;

- $O=$ é o valor do Opex (custo operacional) por barril;

- $Q=$ é a qualidade do óleo expressa pelo grau API;

- $\varepsilon$ = erro padrão da média prevista pelo modelo de regressão; e

- $\beta_{0}, \beta_{1}, \beta_{2}, \ldots . ., \beta_{\mathrm{N}}=$ são os coeficientes do modelo de regressão.
Tabela 2. Cenários com reservas isoladas e adicionadas.

\begin{tabular}{ccc}
\hline & Reservas & $\begin{array}{c}\text { Verificação } \\
\text { dos impactos }\end{array}$ \\
\hline $\begin{array}{c}\text { Cenários de } \\
\text { reserva no } \\
\text { campo }\end{array}$ & $\begin{array}{c}\text { Cenários com adição de } \\
\text { reserva de } 250 \mathrm{MMbbl} \\
\text { sobre } 250,500 \mathrm{e}\end{array}$ & $\begin{array}{c}\text { Comparação } \\
\text { dos } \\
\text { indicadores }\end{array}$ \\
\hline
\end{tabular}

1) $250 \mathrm{MMbbl}$

2) $500 \mathrm{MMbbl}$

3) $750 \mathrm{MMbbl}$

5) $250+250=500 \mathrm{MMbbl}$

VPL, PE,

GT (fatia

4) $1000 \mathrm{MMbbl}$ 7) $750+250=1000 \mathrm{MMbbl}$

governamental)

Cenários de preços analisados: 36, 50 e 70 US\$/bbl. Verificação da relação e impacto das variáveis: Preço, Capex, Opex, API sobre PE e VPL, sob condições de risco. Fonte: adaptado de Massuda (2008).

Para se obter os dados necessários para realizar a regressão envolvendo o VPL e PE em função das $\mathrm{N}$ variáveis independentes pode-se empregar simulação das variáveis incertas por meio do método de Monte Carlo. A partir da geração de N valores aleatórios das variáveis básicas do fluxo de caixa (preço, custo, produção etc.) podem ser obtidas as distribuições de frequências das variáveis de interesse como o VPL e a PE. Essa abordagem denominada de metamodelagem é utilizada em diversos trabalhos na área de E\&P de petróleo, dentre os quais destacam-se Madeira (2005), Risso, Risso e Schiozer (2006) e Avansi et al. (2009). Já na área de análise econômico-estratégica, Kaiser e Pulsipher (2004, 2005) e Massuda (2008) utilizaram essa abordagem para identificar as variáveis independentes que possuem impactos mais críticos sobre as variáveis-respostas VPL e PE. Na Figura 2 encontra-se uma síntese dos principais passos da metodologia de estimativa utilizada.

\section{Aplicação da metodologia aos diversos tipos de cenários de reservas e preços}

Para ilustrar os resultados da metodologia desenvolvida neste artigo foi selecionado um conjunto de reservas típicas das bacias brasileiras. $\mathrm{Na}$ Tabela 3 encontram-se os resultados de PE e VPL para um preço de US\$36/bbl considerando-se dois casos: i) lavra da reserva "sem" módulos adicionais; e ii) lavra da reserva "com" módulos adicionais.

Ressalta-se que para encontrar os valores da Tabela 3 foram consideradas as seguintes premissas para concessões com adição de reservas: 1) defasagem de 10 anos entre os módulos; e 2) os investimentos para a produção dos módulos adicionais são realizados no início da produção do campo. A Tabela 3 deve ser interpretada como segue. 

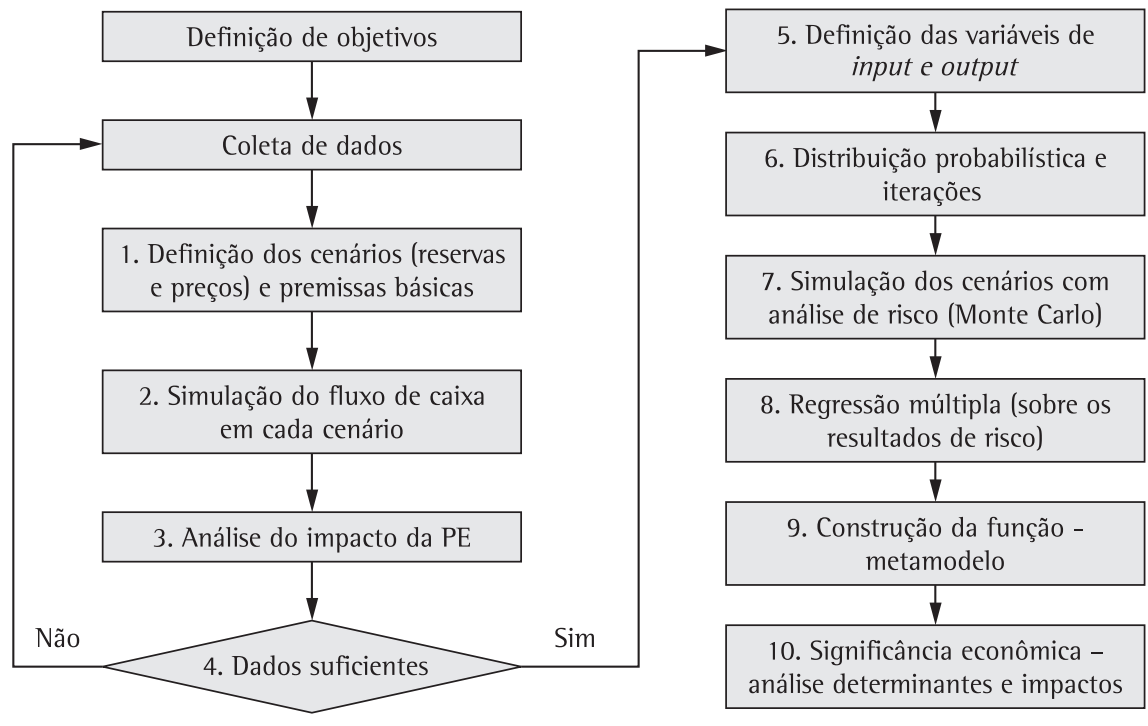

Figura 2. Principais etapas da metodologia da estimativa da PE sobre o VPL do projeto. Fonte: Massuda (2008).

Tabela 3. Resultados de valor presente da PE e VPL com preço de referência de 36,00 US\$/bbl.

\begin{tabular}{cccc}
\hline Cenários & $\begin{array}{c}\text { Reserva } \\
\text { (MMbbl) }\end{array}$ & $\begin{array}{c}\text { Valor } \\
\text { presente } \\
\text { da PE } \\
\text { (MM US\$) }\end{array}$ & $\begin{array}{c}\text { VPL } \\
\text { (MM US\$) }\end{array}$ \\
\hline$(1)$ & 250 & 50,94 & 465,24 \\
$(2)$ & 500 & 302,36 & 329,15 \\
$(3)$ & 750 & 690,77 & 333,47 \\
$(4)$ & 1000 & $1.132,18$ & 302,39 \\
$(5)$ & $250+250=>500$ & 113,29 & $-292,09$ \\
$(6)$ & $500+250=>750$ & 340,46 & $-442,22$ \\
\hline
\end{tabular}

Onde:

-Reserva: refere-se ao volume de óleo, em milhões de barris, lavrados de uma única só vez ou em partes (módulos).

-Valor presente da PE: refere-se ao valor presente de participação especial.

-VPL (valor presente líquido): representa a parcela da empresa. 0 VPL refere-se à somatória dos fluxos de caixa anuais do projeto e é um dos principais indicadores utilizado na indústria de petróleo para análise de decisão.

Fonte: Massuda (2008)

Nos cenários (1) a (4) encontram-se os valores de PE e VPL para concessões com diferentes tamanhos de reservas previamente conhecidas, explotadas (produzidas) com estratégia de produção em um único módulo, o que permite instalação de toda a infraestrutura necessária de uma só vez.

Nos cenários (5) e (6) encontram-se as alternativas de produção em módulos. (A "modularização" pode ocorrer por comprovação de novas reservas a serem lavradas posteriormente, devido aos altos investimentos, por incertezas, por priorização de projetos, entre outros).

Os resultados de VPL e PE obtidos em função do tamanho da reserva apresentaram algumas características interessantes em virtude da estrutura e das peculiaridades do sistema tributário brasileiro.

Para reservas individuais, à medida que se aumenta o volume da reserva nota-se que o valor da PE tende a aumentar, mas no caso do VPL verifica-se que há redução. Por que isso ocorre? Significa que para o investidor é melhor selecionar uma reserva de 250 milhões a outra de 1 bilhão de barris? Faz sentido dizer que o valor de uma reserva de 250 milhões é superior ao de outra reserva cujo volume seja quatro vezes superior? A resposta a essas perguntas repousa em duas variáveis: i) o preço do petróleo; e ii) o comportamento da incidência da PE em termos de suas alíquotas.

Para o caso do preço em US\$36/bbl, a redução do VPL com o aumento do volume da reserva se deve totalmente à PE. Uma vez que esta variável incide sobre grandes produções implica que o aumento da reserva significa maior valor de PE. Dessa forma, o aumento da PE faz com que o $\mathrm{VPL}^{55}$ seja reduzido como mostra a Tabela 3.

$\mathrm{Na}$ prática, pode-se inferir que ao escolher entre pequenos e grandes campos, os investidores preferem os pequenos por diversas razões: i) maior VPL; ii) menor investimento; e iii) menor risco. Para investir em grandes reservas os requisitos de investimentos são significativos e, por isso, o risco envolvido é maior (SUSLICK; SCHIOZER, 2004).

\footnotetext{
${ }^{5}$ Deve-se notar que o VPL depende de outras variáveis como o custo de produção, bem como dos demais tributos. Ao realizar a comparação do VPL e PE, envolvendo diferentes campos, devem ser consideradas as possibilidades de diferentes estruturas de custo. Porém, para os objetivos deste artigo as estruturas de custos foram semelhantes para analisar a sensibilidade dos resultados sob variação das reservas e preços.
} 
Portanto, o retorno do investimento em grandes reservas deve ser superior ao caso de pequenas reservas, mas isso não se verifica nos casos analisados por Massuda (2008). Dessa forma, essa participação governamental pode criar uma barreira contra um dos princípios mais sólidos em finanças: quanto maior o risco, maior deve ser o retorno.

No caso do comportamento da Tabela 3, as alíquotas da PE, quando passam de uma faixa de produção para outra, oscilam entre 0\% até 40\% sobre a receita líquida da produção. Uma vez que o segundo projeto entra em operação após o $4^{\circ}$ ano de produção contado a partir do primeiro módulo, isso faz com que a produção total seja elevada. Se o segundo projeto fosse lavrado isoladamente ele não seria objeto de incidência da PE, devido a sua baixa curva de produção (PE $=0 \%)$. No entanto, assim que a sua produção for adicionada à do primeiro módulo, a curva de produção resultante se torna elevada. Uma vez que para fins de PE considera-se a produção total do ano corrente e a data de início de produção se refere ao primeiro módulo, o resultado é que a alíquota de PE pode ser máxima em 40\% e, por isso, o valor cobrado será elevado e o VPL resultante será reduzido.

É importante observar que essa forma de incidência da PE pode comprometer o projeto e não necessariamente aumentar a arrecadação do governo, no caso de os preços situarem-se nos patamares simulados de US\$36,00. Isso reflete um impacto de $11 \%$ a $374 \%$ de PE maior que o VPL para o modelo estudado.

No caso do desenvolvimento por módulos, os campos pagam maiores alíquotas de PE uma vez que as curvas de produção são somadas. No entanto, os valores presentes de todas as saídas de caixa para pagamento de PE são inferiores, no caso de desenvolvimento por módulos. Por razão similar, o VPL dos projetos desenvolvidos por módulos é inferior, uma vez que fluxos de caixa distantes no futuro são descontados a valor presente. $\mathrm{Na}$ prática, campos de uma única concessão podem ser penalizados no modelo de produção por módulos, decorrentes das ineficiências e assimetrias do modelo fiscal brasileiro.

\subsection{Análise das fatias da empresa e governo em campos com adição de novas reservas (produção por módulos)}

Os conceitos de fatia governamental (GT Government Take) e fatia da empresa (CT - Company Take) são muito utilizados na indústria para realizar comparações acerca da atratividade de diferentes governos. Neste artigo, a fatia governamental (GT) é definida por intermédio da seguinte relação:

$G T=\frac{\text { tributos }}{\text { lucro }+ \text { tributos }}$

E a fatia da empresa é definida pela seguinte relação:

$C T=\frac{\text { lucro }}{\text { lucro }+ \text { tributos }}$

É importante observar que nas definições de GT e CT consideram-se somente duas variáveis: os valores de lucros e de tributos. A outra parcela do fluxo de caixa, que é composta pelo custo total, é excluída, pois esta variável é empregada para sustentar as atividades. 0 objetivo dos conceitos de CT e GT é analisar a intensidade do impacto da estrutura tributária na lucratividade dos projetos e, assim, medir a competitividade dos sistemas tributários entre diferentes paises para auxiliar o processo decisório dos investidores.

Na Figura 3 encontra-se uma análise comparativa dos percentuais da fatia governamental para diferentes tamanhos de reservas e valores de preços.

Para estimar a fatia governamental (GT) da Figura 3, os fluxos de caixa foram atualizados considerando-se uma taxa de desconto igual a 10\%. No caso em que há adição de novas reservas aos campos já em produção, nota-se que à medida que aumenta o preço, a fatia governamental é reduzida. Isso significa que o aumento do preço faz com que ocorra um aumento relativo do valor presente do lucro em relação ao valor presente dos tributos.

Observa-se que a fatia do governo tende a aumentar com a elevação do tamanho da reserva. Isso se deve, especialmente, ao aumento da participação da $\mathrm{PE}$, entre outros tributos. No entanto, ressalta-se que, para todos os níveis de preços, a fatia governamental tende a se estabilizar com o aumento da reserva, crescendo a patamares cada vez menores. Já no caso do aumento de preços, para uma mesma quantidade de lavra, embora a fatia governamental sofra queda, em termos absolutos, sua arrecadação cresce a patamares superiores ao do aumento da reserva. Isto é, a arrecadação de tributos, em valores absolutos, se beneficia mais com um aumento de preços do que com o aumento da reserva.

Uma das principais críticas a esse modelo é que uma vez que muitos dos tributos incidem sobre a produção (PE e royalties), isso implica que nem sempre os campos serão lavrados eficientemente sob o ponto de vista de maximizar o aproveitamento do recurso mineral. 
Government Take\%@10\%, sem e com adição de reserva

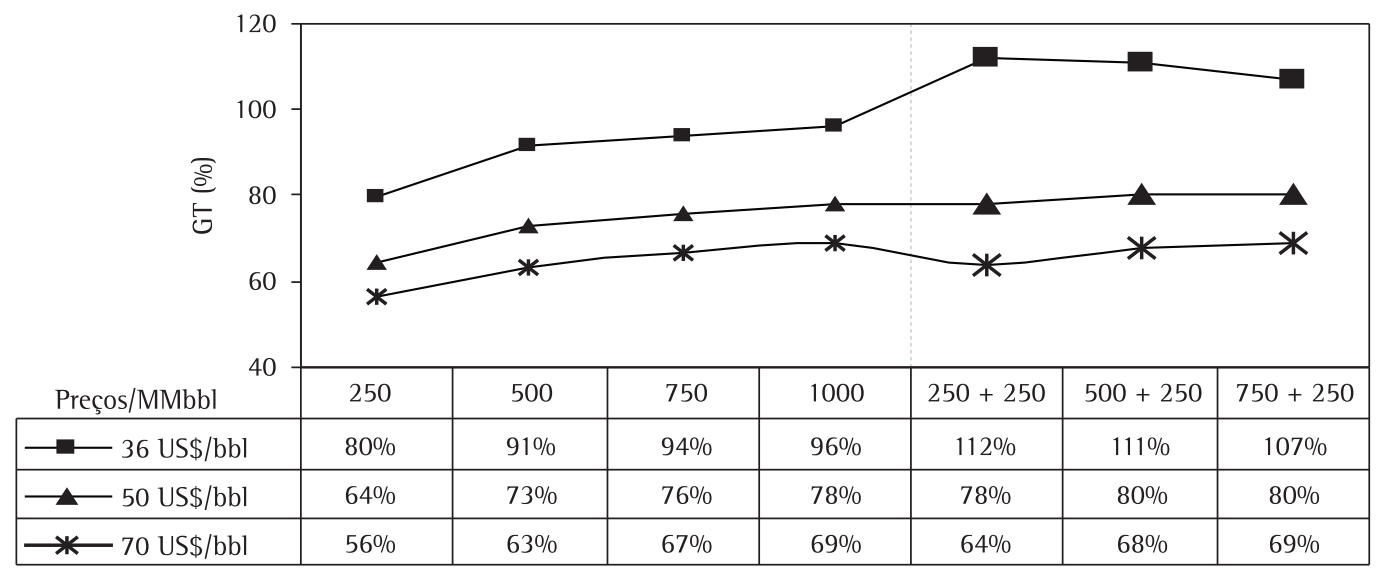

Figura 3. Análise comparativa da fatia do governo (GT) em relação ao preço e tamanho do campo, sem e com adição de reservas. Fonte: adaptado de Massuda (2008).

Ainda na Figura 3 nota-se que em alguns casos a fatia governamental é superior a 100\%. Isso pode acontecer sempre que o lucro for negativo, tal como acontece no caso de campos com adição de reservas.

Os resultados encontrados neste estudo são semelhantes àqueles realizados por Barbosa $\mathrm{e}$ Bastos (2001). Esses autores encontraram resultados análogos em cenários de alto investimento com baixos preços. Com o aumento dos níveis de preços (50 ou 70 US\$/bbl), a fatia do governo aumenta, porém o VPL ainda permanece positivo.

Nos casos em que a fatia governamental for acima de 100\% significa que o VPL é negativo e isso se deve, em parte, às distorções inseridas pela PE em termos de alíquota e base de cálculo. Essa constatação é mais uma manifestação das distorções da base de cálculo da PE que recai sobre a produção e não propriamente sobre a rentabilidade do projeto.

$\mathrm{Na}$ Figura 4 encontra-se uma ilustração da distorção do aumento de alíquota de PE para uma mesma reserva e dois casos: i) um campo com produção isolada de $250 \mathrm{MMbbl}$; ii) quando a produção ocorre pela adição de novas reservas de 250 MMbbl sobre outros campos já em produção de 250, 500 e $750 \mathrm{MMbbl}$.

Alguns comentários sobre as alíquotas da PE mostradas na Figura 4:

- Para o primeiro e segundo ano, uma reserva de 250 milhões de barris possui uma curva de produção, na qual não ocorre incidência da PE (0\%). No terceiro ano $10 \%$, atinge $20 \%$ de alíquota no quarto ano e depois cai para 10\% até a exaustão do campo.
Aliquotas de PE em reserva de $250 \mathrm{MMbbl}$, e quando esta mesma reserva é adicionada tardiamente sobre outra reserva já em produção
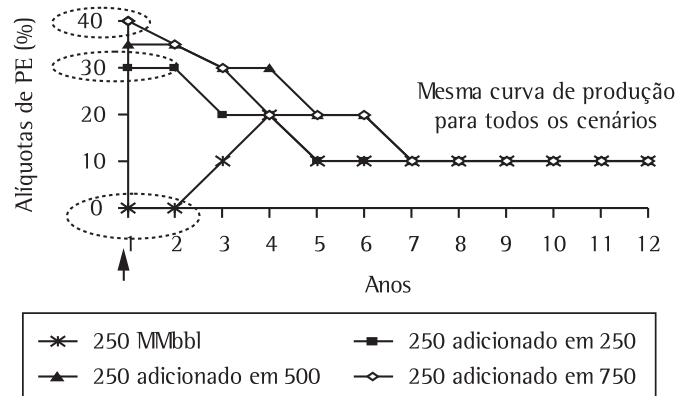

Figura 4. Alíquotas da PE em função da opção de produção do campo com ou sem módulos. Fonte: elaboração própria com base em dados de Massuda (2008).

- À medida que é adicionado um volume de reserva de 250 milhões, as curvas de produção são somadas e as alíquotas crescem. Por exemplo, com apenas uma adição de reserva sobre outra de mesmo tamanho, a alíquota da PE já começa com $30 \%$ no primeiro ano, e somente cai a $10 \%$ a partir do sétimo ano.

- Para o caso de reservas de 750 milhões de barris com adição de 250 milhões, a alíquota no primeiro ano já se inicia com $40 \%$ e somente cai a $10 \%$ a partir do sétimo ano.

De uma forma geral, nota-se que as distorções geradas pela forma de cálculo da PE ocorrem entre os primeiros seis anos de produção, pois a partir do sétimo ano as alíquotas são iguais, independente se a produção ocorre em módulos ou isoladamente. 
Tabela 4. Resultados das fatias do governo e empresas no caso de produção isolada ou em módulos.

\begin{tabular}{|c|c|c|c|c|c|c|c|c|c|}
\hline \multirow{2}{*}{$\begin{array}{l}\text { Reserva } \\
\text { MMbbl }\end{array}$} & \multicolumn{3}{|c|}{ Preço 36 US\$/bbl } & \multicolumn{3}{|c|}{ Preço 50 US\$/bbl } & \multicolumn{3}{|c|}{ Preço 70 US\$/bbl } \\
\hline & $\begin{array}{c}\text { \%GT sem PE } \\
(\%)\end{array}$ & $\begin{array}{c}\% \mathrm{PE} \\
(\%)\end{array}$ & $\begin{array}{r}\% \mathrm{CT} \\
(\%)\end{array}$ & $\begin{array}{c}\% \text { GT sem PE } \\
(\%)\end{array}$ & $\begin{array}{c}\% \mathrm{PE} \\
(\%)\end{array}$ & $\begin{array}{l}\% \mathrm{CT} \\
(\%)\end{array}$ & $\begin{array}{c}\% \text { GT sem PE } \\
(\%)\end{array}$ & $\begin{array}{l}\% \mathrm{PE} \\
(\%)\end{array}$ & $\begin{array}{r}\% \mathrm{CT} \\
(\%)\end{array}$ \\
\hline 250 & 77 & 2 & 20 & 62 & 2 & 36 & 54 & 2 & 44 \\
\hline 500 & 83 & 8 & 9 & 63 & 9 & 27 & 53 & 10 & 37 \\
\hline 750 & 82 & 12 & 6 & 62 & 14 & 24 & 52 & 15 & 33 \\
\hline 1000 & 81 & 15 & 4 & 60 & 17 & 22 & 50 & 18 & 31 \\
\hline \multicolumn{10}{|c|}{ Cenários com adição de reserva de $250 \mathrm{MMbbl}$ (segundo módulo) (\%) } \\
\hline $250+250$ & 108 & 5 & -12 & 73 & 5 & 22 & 59 & 5 & 36 \\
\hline $500+250$ & 103 & 8 & -11 & 70 & 10 & 20 & 56 & 11 & 32 \\
\hline $750+250$ & 96 & 11 & -7 & 67 & 14 & 20 & 54 & 15 & 31 \\
\hline
\end{tabular}

Fonte: adaptado de Massuda (2008).

\subsection{Análise da fatia da empresa, do governo e o impacto da participação especial}

$\mathrm{Na}$ Tabela 4 encontra-se uma análise dos percentuais da fatia governamental e da fatia da empresa, bem como os impactos da PE nessas duas varáveis mencionadas.

Para estimar os valores que se encontram na Tabela 4 foram considerados os valores presentes de todos os fluxos de caixa. Nota-se que para a reserva de $250 \mathrm{MMbbl}$ ao preço de $36 \mathrm{US} \$ / \mathrm{bbl}$, o percentual da fatia do governo (GT sem PE) foi de 77\% com ligeiro acréscimo (83\%) quando duplica-se as reservas (500 MMbbl). Enquanto isso a PE subiu de $2 \%$ a $15 \%$ de acordo com o tamanho da reserva, e a fatia da empresa (CT) teve queda de $20 \%$ para $4 \%$. Portanto, a redução da fatia da empresa (CT) ocorre em função do aumento da PE.

Além disso, a Tabela 4 permite verificar que o aumento da fatia do governo ocorreu em função do aumento da participação especial. Esse comportamento pode ser percebido comparando-se as colunas "GT sem PE" (que indica a fatia do governo sem a participação especial), "PE" (participação especial) e "CT" (fatia da empresa).

Para os cenários com adição de reserva há diferenças importantes nos indicadores entre uma reserva de $500 \mathrm{MMbbl}$ e outra reserva de $250 \mathrm{MMbbl}$ com adição de mais $250 \mathrm{MMbbl}$ que, embora detenham os mesmos volumes de $500 \mathrm{MMbbl}$, devido à metodologia de cálculo da PE: a fatia do governo (GT) cresce de $83 \%$ para 108\%, enquanto a PE decresce de $8 \%$ para $5 \%$ e a fatia da empresa (CT) apresenta uma queda de 9\% para $-12 \%$.

Para todos os cenários com adição de reserva, ao preço de 36 US\$/bbl, o GT fica acima de 100\%, inviabilizando o projeto. Nas simulações com preços mais elevados (50 e 70 US $\$ /$ bbl) o GT cresce, entretanto a PE e o CT sofrem menor impacto.
Tabela 5. Comparação da razão PE sobre GT para campos isolados e com adição de reserva.

\begin{tabular}{|c|c|c|c|}
\hline \multirow{2}{*}{$\begin{array}{l}\text { Reserva } \\
\text { MMbbl }\end{array}$} & 36 US\$/bbl & 50 US\$/bbl & $70 \mathrm{US} \$ / \mathrm{bbl}$ \\
\hline & $\% \mathrm{PE} / \mathrm{GT}(\%)$ & $\% \mathrm{PE} / \mathrm{GT}(\%)$ & \%PE/GT (\%) \\
\hline 250 & 3 & 3 & 4 \\
\hline 500 & 9 & 13 & 15 \\
\hline 750 & 13 & 19 & 22 \\
\hline 1000 & 15 & 22 & 27 \\
\hline \multicolumn{4}{|c|}{ Cenários com adição de reserva de $250 \mathrm{MMbbl}$} \\
\hline $250+250$ & 4 & 6 & 8 \\
\hline $500+250$ & 8 & 13 & 17 \\
\hline $750+250$ & 10 & 17 & 22 \\
\hline
\end{tabular}

PE: Participação especial; GT: Fatia do governo. Fonte: Massuda (2008).

Esses resultados demonstram que há distorções no cálculo da participação especial, que não é linear e não considera a existência de vários projetos em um só campo. Se os preços estiverem mais elevados, o impacto é minimizado, porém os efeitos da não linearidade e da rentabilidade não são incluídos pela forma atual de incidência da PE.

$\mathrm{Na}$ Tabela 5 encontra-se uma análise envolvendo $\% \mathrm{PE} / \mathrm{GT}$ (percentual da PE sobre a fatia do governo) para os diferentes tamanhos de reservas produzidas isoladas ou em módulos.

Observa-se que o aumento da fatia de PE não é linear, proporcional à produção, e seu crescimento é maior para maiores níveis de preços e de reserva. Portanto, quanto maior o campo e os preços, maior a arrecadação de participação especial, se mantidas as demais variáveis constantes. Pereira (2004) e Gandra (2006) constaram a mesma tendência de crescimento do peso da PE sobre o GT de acordo com o aumento do tamanho da reserva. Em relação à adição de reservas, para projetos em módulos, a relação da PE sobre o GT decresce para todos os casos de reserva e níveis de preços aqui analisados. 
Tabela 6. Coeficientes do metamodelo da PE e do VPL para diferentes reservas e cenários de preços.

\begin{tabular}{|c|c|c|c|c|c|c|c|c|}
\hline \multicolumn{9}{|c|}{ PE } \\
\hline Reserva & \multicolumn{2}{|c|}{$250 \mathrm{MMbbl}$} & \multicolumn{2}{|c|}{$500 \mathrm{MMbbl}$} & \multicolumn{2}{|c|}{$750 \mathrm{MMbbl}$} & \multicolumn{2}{|c|}{$1000 \mathrm{MMbbl}$} \\
\hline Preço & 36 & 50 & 36 & 50 & 36 & 50 & 36 & 50 \\
\hline$\beta_{1}$ & $-98,24$ & $-131,80$ & $-1.720,24$ & $-1.098,97$ & $-1.757,10$ & $-2.483,68$ & $-727,27$ & $-4.175,77$ \\
\hline$\beta_{2} P$ & 2,69 & 2,61 & 47,28 & 21,49 & 47,61 & 49,56 & 20,80 & 81,62 \\
\hline$\beta_{3} C$ & $-5,97$ & $-5,19$ & $-122,57$ & $-57,33$ & $-123,18$ & 137,44 & $-54,97$ & $-227,26$ \\
\hline$\beta_{4} 0$ & $-5,64$ & $-5,61$ & $-94,74$ & $-49,18$ & $-96,15$ & 106,52 & $-40,14$ & $-181,65$ \\
\hline$\beta_{5} Q$ & 2,72 & 3,65 & 47,74 & 30,90 & 48,54 & 70,02 & 20,13 & $-118,02$ \\
\hline $\mathrm{R}^{2}$ & 0,98 & 0,99 & 0,97 & 0,99 & 0,97 & 0,99 & 0,97 & 0,99 \\
\hline \multicolumn{9}{|c|}{ VPL } \\
\hline Reserva & \multicolumn{2}{|c|}{$250 \mathrm{MMbbl}$} & \multicolumn{2}{|c|}{$500 \mathrm{MMbbl}$} & \multicolumn{2}{|c|}{$750 \mathrm{MMbbl}$} & \multicolumn{2}{|c|}{$1000 \mathrm{MMbbl}$} \\
\hline Preço & 36 & 50 & 36 & 50 & 36 & 50 & 36 & 50 \\
\hline$\beta_{1}$ & $-2.504,20$ & $-3.395,70$ & $-5.952,67$ & $-5.576,30$ & $-6.030,60$ & $-7.924,39$ & $-4.244,80$ & $-10.010,80$ \\
\hline$\beta_{2} P$ & 68,68 & 66,85 & 164,83 & 111,83 & 164,69 & 156,73 & 117,07 & 198,99 \\
\hline$\beta_{3} C$ & $-286,73$ & $-275,39$ & $-840,42$ & $-540,41$ & $-835,26$ & $-776,03$ & $-573,81$ & $-1.001,24$ \\
\hline$\beta_{4} 0$ & $-154,69$ & $-150,09$ & $-408,05$ & $-271,00$ & $-407,38$ & $-382,22$ & $-287,51$ & $-481,72$ \\
\hline$\beta_{5} Q$ & 69,85 & 94,83 & 168,34 & 156,21 & 169,79 & 220,82 & 119,19 & 277,38 \\
\hline $\mathrm{R}^{2}$ & 0,99 & 0,99 & 0,99 & 0,99 & 0,99 & 0,99 & 0,99 & 0,99 \\
\hline
\end{tabular}

Observações: os valores de P -value são todos os interceptos e coeficientes angulares são estatisticamente significativos no nível de 1\%; os valores de R2 são todos acima de 97\%, mostrando que somente 3\% da variabilidade de PE e VPL em todos de suas médias se devem a outros fatores. Fonte: Massuda (2008).

Porém, a queda é mais reduzida para níveis de preços mais elevados.

\section{Análise do impacto de variáveis incertas na PE e no VPL}

Para estimar o impacto das diversas variáveis de entrada nas variáveis de saída, que são o VPL e PE, foi realizada uma simulação de Monte Carlo para que se pudesse desenvolver uma regressão entre os valores das variáveis de entrada (input) e os das variáveis de saída (output). Essa abordagem, que consiste em realizar regressões a partir de dados gerados por simulação, é conhecida por metamodelagem. Assim, é possível quantificar a sensibilidade das variáveis de saída em relação às variáveis de entrada do modelo.

Foram considerados oito cenários compostos por diferentes combinações de preço e tamanho de reservas, os quais se encontram na Tabela 6.

A partir desses resultados, foram construídas as equações de PE e VPL em função das variáveis consideradas no presente estudo. Por exemplo, para reservas de 250 milhões de barris e preço de US\$ 50/bbl, a equação será:

$\mathrm{VPL}=-3.395,7+66,85^{*} \mathrm{P}-275,09^{*} \mathrm{C}-$

$-150,09^{*} 0+94,83^{*} \mathrm{Q}+\mathrm{e}$,
$\mathrm{PE}=-131,8+2,61^{*} \mathrm{P}-5,19^{*} \mathrm{C}-5,61^{*} \mathrm{O}+$

$+3,65^{*} \mathrm{Q}+\mathrm{e}$,

onde "e" é o erro padrão da regressão. Assim, por exemplo, o aumento de uma unidade do preço (P) implica aumento de 66,85 unidades no VPL e de 2,61 unidades na PE.

Pode-se observar que para a PE a variável mais crítica (fora o intercepto) é o custo operacional "opex" (0), seguido pelos investimentos (C) e qualidade do óleo (0). Já para o VPL, a variável mais significativa são os custos com investimentos "capex" (C), ou seja, o aumento de uma unidade sobre os investimentos implicam em redução em 275,09 unidades no VPL. As demais equações (metamodelos) constantes da Tabela 6 têm interpretação análoga ao caso anterior e servem como ferramenta para planejamento, tanto das empresas quanto dos organismos reguladores.

\section{Conclusões}

Este artigo demonstrou os resultados do estudo sobre a participação especial (PE), seus principais determinantes e impactos econômicos em função de tamanho de reserva e da lavra isolada ou em módulos, com adição de reservas ao longo do tempo. Os principais aspectos indicados nas simulações são: 
A PE provoca mais impactos nos indicadores econômicos quando a produção é desenvolvida em módulos e quando os módulos adicionais (adição de reserva) de uma mesma área de concessão ocorrem antes do sétimo ano do início da produção do primeiro módulo;

Conforme cresce o tamanho das reservas, o valor da PE aumenta enquanto o VPL por barril pode decrescer. Esse comportamento pode ocasionar distorções na relação risco/retorno, pois os investimentos em exploração e produção sempre se baseiam em fundamentos tradicionais utilizados na tomada de decisão de "quanto maior o risco, maior deve ser o retorno";

Com o aumento do preço do petróleo, a fatia governamental tende a diminuir exatamente porque cresce o lucro da empresa (porém, em valores absolutos, a arrecadação do governo permanece crescente);

À medida que aumenta o preço do petróleo, os impactos da PE, devido à adição de novos módulos aos que já se encontram em produção, se tornam cada vez menos significativos, conforme se aumenta o tamanho da reserva.

Como a produção em módulos é característica de grandes reservas, devido aos altos riscos e investimentos envolvidos, tanto os campos maduros do pós-sal como os novos grandes campos do pré-sal estão sujeitos a esse impacto.

Porém, ainda mais importante que os impactos econômicos da PE em investimentos individualizados são as distorções sistêmicas, aumentando a resistência das empresas a investirem nas atividades de exploração e produção de petróleo, com o agravante de o governo não necessariamente estar arrecadando mais.

\section{Referências}

AGÊNCIA NACIONAL DO PETRÓLEO, GÁS NATURAL E BIOCOMBUSTÍVEIS (Brasil). Dados Estatísticos. 2008. Disponivel em: <http://www.anp.gov.br/>. Acesso em: 15 dez. 2008.

AGÊNCIA NACIONAL DO PETRÓLEO, GÁS NATURAL E BIOCOMBUSTÍVEIS (Brasil). Legislação. 2009. Disponível em: <http://www.anp.gov.br/>. Acesso em: 15 jan. 2009.

AVANSI, G. et al. Assisted Procedures for Definition of Production Strategy and Economic Evaluation Using Proxy Models. In: SPE EUROPEC/EAGE ANNUAL CONFERENCE AND EXHIBITION, 2009. Proceedings... Amsterdam: Society of Petroleum Engineers, 2009. 9 p. http://dx.doi.org/10.2118/122298-MS

BARBOSA, D. H.; BASTOS, A. C. Impacto da Tributação nas Atividades de E\&P em Águas Profundas no Brasil. In Suslick, S. (Org.). Regulação em Petróleo e Gás Natural. Campinas: Editora Komedi, 2001. p.48-103.
BRASIL. Agência Nacional do Petróleo, Gás Natural e Biocombustíveis. Portaria ANP № 10, de 13 de janeiro de 1999. Estabelece os procedimentos para a apuração, pelos concessionários das atividades de produção de petróleo, gás natural ou ambos, da participação especial prevista no art. 50 da Lei $n^{\circ} 9.478$, de 6 de agosto de 1997, em complementação ao Capítulo VII do Decreto $n^{\circ} 2.705$, de 3 de agosto de 1998. Diário Oficial da República Federativa do Brasil, Agência Nacional do Petróleo, Brasília, DF, 13 jan. 1999.

BRASIL. Decreto No 2.705, de 3 de agosto de 1998. Define critérios para cálculo e cobrança das participações governamentais de que se trata esta Lei, aplicáveis às atividades de exploração, desenvolvimento e produção de petróleo e gás natural. Diário Oficial da República Federativa do Brasil, Poder Executivo, Brasília, DF, 04 ago. 1998.

BRASIL. Lei № 9.478, de 6 de agosto de 1997. Dispõe sobre a política energética nacional, as atividades relativas ao monopólio do petróleo, institui o Conselho Nacional de Política Energética e a Agência Nacional do Petróleo e dá outras providências. Diário Oficial da República Federativa do Brasil, Poder Executivo, Brasília, DF, 07 ago. 1997.

FRASER, R. An evaluation of the relative performance of alternatively structured resource rent taxes. Resources Policy, v. 28, p. 1-6, 2002. http://dx.doi.org/10.1016/ S0301-4207(02)00012-0

GAMPONIA, V.; MENDELSOHN, R. The Taxation of Exhaustible Resources. The Quarterly Journal of Economics, v. 100, n. 1, p. 165-181, 1985. http://dx.doi. $\operatorname{org} / 10.2307 / 1885740$

GANDRA, R. M. Impacto da participação especial em campos gigantes offshore de petróleo. Produção, v. 16, n. 2, p. 274-286, 2006. http://dx.doi.org/10.1590/S010365132006000200008

KAISER, M. J.; PULSIPHER, A. G. A Meta-Modeling Approach to Fiscal System Analysis, Part 11. Deepwater Royalty Relief in the Gulf of Mexico: A Case Study of Na Kika. Petroleum Acconting and Financial Management Journal, v. 23, n. 3, p. 60, 2004.

KAISER, M. J.; PULSIPHER, A. G. A Meta-Modeling Approach to Fiscal System Analysis, Part Ill. Production Sharing Agreements. Petroleum Acconting and Financial Management Journal, v. 24, n. 1, p. 59, 2005.

MADEIRA, M. G. Comparação de técnicas de análise de risco aplicadas ao desenvolvimento de campos de petróleo. 2005. 132 f. Dissertação (Mestrado em Ciências e Engenharia de Petróleo)-Universidade Estadual de Campinas, Campinas, 2005.

MASSUDA, E. T. O Impacto da participação especial em campos de petróleo marítimos utilizando a Meta-Modelagem. 2008. Dissertação (Mestrado em Geociencias)-Universidade Estadual de Campinas, Campinas, 2008.

NAKHLE, C. Do high oil prices justify an increase in taxation in a mature oil province? The case of the UK continental shelf. Energy Policy, v. 35, p. 4305-4318, 2007. http://dx.doi.org/10.1016/j.enpol.2007.02.021

PEREIRA, M. A. Avaliação do impacto dos tributos na incorporação de reservas nas empresas do setor de petróleo. 2004. 130 f. Dissertação (Mestrado em Ciências em Planejamento Energético)-Universidade Estadual do Rio de Janeiro, Rio de Janeiro, 2004. 
RISSO, V. F.; RISSO, F. V. A.; SCHIOZER, D. J. Ajuste de Histórico de Campos de Petróleo Utilizando a Metodologia do Planejamento Estatístico. In: CONGRESSO IBEROLATINO-AMERICANO DE MÉTODOS COMPUTACIONAIS EM ENGENHARIA - CILAMCE, 27., 2006, Belém, Pará. Anais... Belém: ABMEC, 2006, p. 03-06.

ROCHA, W. S.; RIBEIRO, R. A.; MARQUES, J. A. V. Participações governamentais da indústria do petróleo e gás natural e as demonstrações contábeis. In: CONGRESSO BRASILEIRO DE P\&D EM PETRÓLEO E GÁS - PDPETRO, 4., 2007, Campinas. Anais... Campinas: ABPG, UNICAMP, 2007. p. 21-24.

SUSLICK, S. B. A dinâmica da regulação no setor de petróleo e gás natural. In: SUSLICK, S. B. (Org.) Regulação em petróleo e gás natural. Campinas: Ed. Komedi, 2001. p. 33-45. http://dx.doi.org/10.1016/j.petrol.2004.02.001
SUSLICK, S. B.; SCHIOZER, D. J. Risk Analysis Applied to Petroleum Exploration and Production: An Overview. Journal of Petroleum Science and Engineering, v. 44 n. 2, p. 1-9, 2004.

TILTON, J. E. Determining the optimal tax on mining. Natural Resources Forum, v. 28, p.144-149, 2004. http://dx.doi. $\operatorname{org} / 10.1111 / j .1477-8947.2004 .00081 . x$

\section{Agradecimentos}

Os autores agradecem aos revisores pelos comentários, ao CNPq e ao CEPETRO - Centro de Estudos de Petróleo, da Unicamp - Universidade Estadual de Campinas, pelo apoio financeiro para a realização desta pesquisa.

\title{
Special participation tax: analysis off determinants and economic impacts in the offshore oilfields production projects
}

\begin{abstract}
The special participation fee is used to capture the rent originated from oil production fields with a great production profile, like Pre-salt or brown fields in Campos Basin. Considering the complexity of this fee, in this paper different forms of its impacts in the oilfields projects are considered: a production profile generated by only one production module and by more modules. The methodology combines the cash flow analysis from the projects and NPV estimations, using stochastic simulations. The results obtained from the simulation models indicated inefficiencies generated, both in its form of incidence and rates as well as in its estimation process. This type of distortion occurs for giant oilfields with different characteristics (reserves up to 1 billion barrels, simulation cut-off volumes used in this study) and for those fields with reserves additions. The development of the production field in modules in the same field, the second module is overload, generating potential unfeasibility for the project, if the reference price is not sufficient high.
\end{abstract}

\section{Keywords}

Special participation tax oil. Economic viability. Project. Meta-modeling. 